\title{
rommhalina
}

(8)

\section{La bataille d'Aegitna}

Autor(es): $\quad$ Mathieu-Pavard, Dominique; Thiercy, Pascal

Publicado por: Imprensa da Universidade de Coimbra

URL

persistente:

URI:http://hdl.handle.net/10316.2/38352

DOI:

DOI:http://dx.doi.org/10.14195/978-989-26-0438-1_12

Accessed : $\quad$ 26-Apr-2023 11:48:37

A navegação consulta e descarregamento dos títulos inseridos nas Bibliotecas Digitais UC Digitalis, UC Pombalina e UC Impactum, pressupõem a aceitação plena e sem reservas dos Termos e Condições de Uso destas Bibliotecas Digitais, disponíveis em https://digitalis.uc.pt/pt-pt/termos.

Conforme exposto nos referidos Termos e Condições de Uso, o descarregamento de títulos de acesso restrito requer uma licença válida de autorização devendo o utilizador aceder ao(s) documento(s) a partir de um endereço de IP da instituição detentora da supramencionada licença.

Ao utilizador é apenas permitido o descarregamento para uso pessoal, pelo que o emprego do(s) título(s) descarregado(s) para outro fim, designadamente comercial, carece de autorização do respetivo autor ou editor da obra.

Na medida em que todas as obras da UC Digitalis se encontram protegidas pelo Código do Direito de Autor e Direitos Conexos e demais legislação aplicável, toda a cópia, parcial ou total, deste documento, nos casos em que é legalmente admitida, deverá conter ou fazer-se acompanhar por este aviso. 
Francisco de Oliveira

Pascal Thiercy

Raquel Vilaça

Coordenação

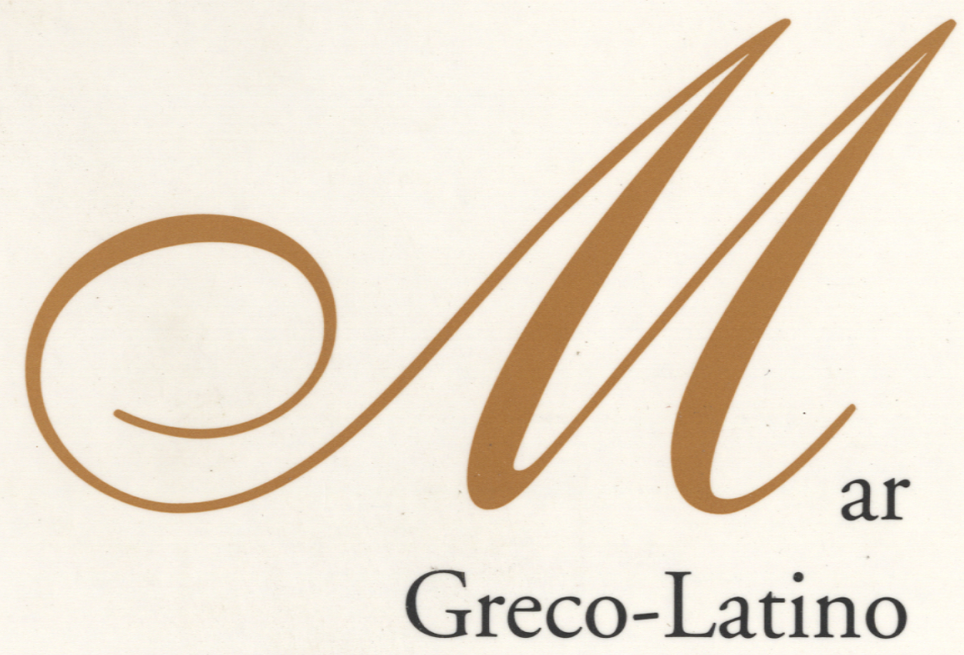

- colmbra 2006 


\section{LA BATAILLE D'AEGITNA}

Dominique Mathieu-Pavard

Pascal Thiercy

Université de Bretagne Occidentale

\section{Les Ligures}

Au IIe siècle avant Jésus-Christ, les Grecs occupaient depuis si longtemps la Côte d'Azur qu'on avait dû oublier quand ils étaient arrivés. Pendant un demi-millénaire, ces Grecs ont voisiné avec les tribus ligures. Vers 1.600 avant J.-C., ces Ligures, venus du N. E. de l'Europe, en colonisent une grande partie. Ce peuple n'a laissé que peu de traces, car il ne connaissait pas l'écriture, mais les géographes grecs et romains ont parlé d'eux. Appelés Capilatti par les Romains, ces Ligures étaient de solides montagnards combatifs, souvent utilisés par la suite comme mercenaires par les Romains qui ont mis 160 ans à les soumettre (de 154 avant J- C., bataille d'Aegitna, à 6 après J - C., trophée d'Auguste), alors que Jules César n'a mis que 9 ans (de 58 à 50 avant J - C.) pour conquérir la Gaule, hors la Narbonnaise. Il nous reste d'eux des enceintes de tailles différentes, de 2 à $4 \mathrm{~m}$., en pierres sèches sans liant de chaux.

Les principales peuplades étaient :

Les Vésubianii, dans la vallée de la Vésubie ; les Suelteri, qui occupaient tout le massif de l'Estérel auquel ils ont donné son nom; les Éburiates 
(Euburiates) peuplaient Monaco et les alentours; les Ligaunes étaient, semble-t-il, répandus entre Auribeau et Grasse ; les Vélaunii, les Nérusiens, les 206 Oratelli, les Veamini, les Ectinii, les Nemeturii, les Eguituri, dans les hautes et moyennes vallées du Var; les Védiantii, entre le Var et La Turbie, dont la métropole était Cimiez, sur les hauteurs de Nice (les Romains en feront la capitale de la province romaine des Alpes-Maritimes en l'an 7 avant J.-C. sous le nom de Cemenelum), et ceux qui nous intéressent principalement aujourd'hui : les Oxybiens, qui habitaient les parages d'Antipolis et avaient pour chef-lieu Aegitna, et les Déciates (ou Décéates), situés également dans l'arrière-pays d'Antipolis (Antibes), quelque part entre la Siagne et le Loup ; leur capitale, Deciatum, aurait pu être les Encourdoules ou l'oppidum d'Antipolis.

L'inscription du trophée d'Auguste de la Turbie énumère les noms de 48 tribus soumises par Rome. Sur cet ensemble, 17 appartiennent à la Côte d'Azur, sans compter les Oxybiens, les Déciates et les Vediantii, vaincus antérieurement, qui n’y figurent pas. Le peuplement de la région devait être particulièrement morcelé, sans doute en raison du relief tourmenté.

Pendant quatre siècles, les Grecs et les indigènes avaient vécu dans une relation de bon voisinage sous les cieux cléments de la Côte d'Azur. Alors, que s'est-il passé au deuxième siècle avant notre ère? Les deux groupes humains vivaient cloisonnés, mais, s'il n'y avait pas fusion, il y avait échanges commerciaux et amicaux. Néanmoins, le passage d'une civilisation à peine sortie du néolithique à une civilisation hellénisée ne se faisait pas sans douleur.

L'avancée de l'hellénisation avait donné naissance à une classe d'indigènes évolués ayant fait fortune en suivant l'exemple des marchands grecs. Inversement, nombre de colons dont les ressources diminuaient du fait du ralentissement du commerce massaliote, se retrouvaient au même niveau que les indigènes aisés. D'où de nouvelles tensions, car les niveaux de vie s'homogénéisant rendent plus sensibles les différences: on ne supporte plus 
les écarts de fortune. Le plan de clivage n'est plus ethnique. Il devient économique et oppose le barbare enrichi au civilisé appauvri. À la phase finale de l'hellénisation, pour la première fois sur la souriante Côte d'Azur, souffle un vent de violence chargé d'aspirations contraires : être ou ne pas être Grec?

Pourquoi donc ce processus de violence entre les Ligures et les Grecs sur la Côte d'Azur au IIe siècle avant notre ère ? On peut avancer quelques pistes :

Explication économique : l'affaiblissement de Massalia

Les indigènes de la Côte d'Azur, qui tiennent les routes et le bord de mer, en profitent pour piller les convois terrestres et les navires massaliotes qui cabotent le long des côtes. Les indigènes avaient toléré, par intérêt commercial, l'édification des comptoirs massaliotes, mais lorsque les colons se mettent à planter leurs ceps dans les sillons ligures et à jeter leurs filets dans leurs eaux, ils voient rouge! Le principal contentieux réside sans doute dans l'extension des colons grecs sur les terres et le littoral ligures. Au IIe siècle av. J.- C., les Ligures de la Côte d'Azur ne sont plus tout à fait des Barbares. Leur essor commercial concurrence sérieusement celui des Grecs et des Romains, sur mer et surtout, sur terre, pour les produits issus du raisin et des olives, l'huile et le vin.

Explication fonctionnaliste : la xénophobie

On met toujours l'économie en avant dans les problèmes qui opposent deux sociétés, mais les sentiments entrent aussi en ligne de compte. Les Grecs jouissaient par rapport aux indigènes d'une situation beaucoup plus enviable. Il pourrait y avoir eu alors une soudaine prise de conscience de l'écart social entre les colons et les Ligures. Les indigènes envient les cités prospères des Grecs : le confort et le luxe dont bénéficient les colons poussent à vouloir les imiter. Les frustrations déclenchent des réactions de violence : les colons grecs 
deviennent les boucs émissaires et le bel enthousiasme d'une civilisation porteuse de progrès se fracasse contre l'impossible entente avec l'autre, le voisin, ce barbare.

Explication " anti-mondialiste "

Les Grecs n'ont pas voulu englober le monde ligure dans leur civilisation, mais ils ont perturbé les usages et les traditions et ont fini par resserrer la cohérence ethnique des indigènes dans un réflexe de protection identitaire. Les Grecs ont apporté aux indigènes la monnaie, la consommation, l'écriture et une langue de communication internationale, le Grec. Ce sont les prémisses d'une sorte de globalisation contre laquelle les indigènes vont s'insurger en véhéments anti-mondialistes avant la lettre.

\section{ROMAINS ET LIGURES}

Pendant les guerres puniques les Romains traversent la région pour combattre les Carthaginois en Espagne de 264 à 146 avant J.-C. La voie Héracléenne, qui suit le bord de mer, était difficile d'accès, aussi le plus gros du transport de troupe se faisait-il par mer avec escale à Monaco, Nice ou Antibes, villes grecques alliées de Rome. Pendant l'intervalle entre la deuxième et la troisième guerre punique (201-149), des peuples alpins, que l'on nomma les Gallo-Ligures, s'allièrent pour défendre leurs territoires, et se groupèrent sur les bords du Var. Des combats les opposèrent aux Romains et ont été relatés notamment par Tite-Live et Paul Orose.

Le premier eut lieu sur la rivière de Gênes, non loin de la Côte d'Azur ; en 197 avant Jésus-Christ, Quintus Minucius Rufus écrase les Ligures révoltés. En 189, Lucius Baebius Dives, un préteur romain qui se dirigeait vers l'Espagne à la tête de quelques légions, s'empara de la ville de Cimiez et entra dans Nice 
après avoir passé le Var. Les Gallo-Ligures profitèrent de la nuit pour massacrer Baebius et tous ses soldats.

Paul Orose raconte ainsi cet épisode : « Laelius Baebius, partant pour l'Espagne, fut enveloppé par les Ligures et tué avec ses soldats dont pas un ne survécut, de sorte que la nouvelle de ce désastre fut envoyée à Rome par les habitants de Marseille, alliés des Romains ${ }^{(1)}$ ".

Selon Tite-Live, il parvint à se traîner jusqu'à Massalia pour y mourir, trois jours après son arrivée ${ }^{(2)}$ : Per eos dies, quibus baec gesta sunt, legati Massiliensium nuntiarunt L. Baebium Praetorem, in provinciam Hispaniam proficiscentem, ab Liguribus circumventum magna parte comitum caesa vulneratum ipsum cum paucis sine lictoribus Massaliam perfugisse et intra triduum expirasse.

Orose raconte ensuite que quelque temps après, les mêmes peuplades gallo-liguriennes livrèrent encore bataille, avec le même succès, au consul Quintus Marcius, envoyé pour venger la mort de Baebius, et tuèrent quatre mille hommes; lui-même aurait péri s'il n'avait battu en retraite pour se réfugier dans son camp: Q. Marcius, consul adversus Liguras profectus superatusque, quatuor millia militum amisit et, nisi victus celeritate refugisset in castra, eamdem internecionis cladem quam Baebius ab eisdem hostibus acceperat pertulisset.

En l'an 181, le consul Baebius Tamphilus réprime un soulèvement massif des Ligures Apuani. 40000 prisonniers sont déportés dans le Samnium. La même année, les Ingaunes établis entre Albenga et Vintimille, à la frontière de la Côte d'Azur, s'en prennent violemment aux bateaux massaliotes qui cabotent le long des rivages. Rome dépêche le consul L. Aemilius Paulus. Aux dires de Plutarque ${ }^{(3)}$, les trois quarts des Ingaunes sont massacrés, les

\footnotetext{
(1) Loelius Boebius, in Hispaniam proficiscens, a Liguribus circumventus, cum universo exercitu occisus est; unde adeo ne unum quidem superfuisse constat, ut internecionem ipsam Romoe, Massilienses nuntiare curaverint. (Lib.IV, 20)

(2) Tite-Live, XXXII, 29, 6.

(3) Plutarque, Paul Émile, VI, a.
} 
survivants sont emmenés en esclavage. Paul Émile détruit les remparts, les récoltes et les vignes, n'épargnant que les femmes et les enfants, et interdit la fabrication d'armement et d'embarcations de plus de trois avirons. Une flotte romaine assure la police sur les côtes de la Ligurie. Les tribus indigènes abandonnent la piraterie et se reconvertissent dans le banditisme de grand chemin, imitant les tribus de la Côte d'Azur.

En 154 avant Jésus-Christ, les Oxybiens et les Déciates s'agitent dangereusement. Leurs premières visées sont Antipolis et Nikaïa. La chute des principaux comptoirs grecs pourrait avoir un effet catastrophique sur le moral des Massaliotes, déséquilibrer l'économie maritime et entraver la circulation d'Italie à l'Espagne.

Moment fort de la conquête romaine, Aegitna, en 154 avant Jésus-Christ, a été le Waterloo de la Côte d'Azur. La bataille opposant les indigènes aux Romains a été perdue pour un problème de retard dans l'arrivée des renforts. Comme Napoléon guettant la venue de Grouchy dans la mêlée effroyable et vivante broussaille, les Oxybiens attendaient les Déciates: pour Napoléon, ce fut Blücher et pour les Oxybiens... les Romains ! L'espoir changea de camp, le combat changea d'âme et les barbares s'effondrèrent comme un fleuve. Ce fleuve, c'était l'Apron, et pour les Oxybiens, ce fut une vraie Bérézina...

Polybe, dans ses Histoires ${ }^{(4)}$, consacre un récit circonstancié à cet événement riche en action et en rebondissements. À cette période, il résidait à Rome, chez les Scipion, et a dû en recueillir le récit auprès de témoins directs. Le livre XLVII de Tite-Live, qui relate les mêmes faits, a malheureusement disparu. Le récit de Polybe est le seul à avoir survécu, mais le texte que nous possédons, relatant la bataille d'Aegitna, est incomplet. Il provient d'une compilation réalisée au Xe siècle sur la demande du Basileus byzantin Constantin VII Porphyrogénète.

\footnotetext{
(4) Polybe, Histoires, fragments du livre XXXIII, 8-11, traductions de P. Thiercy.
} 


\section{AEGITNA : L'INSULTE FAITE AUX CONSULS}

“O

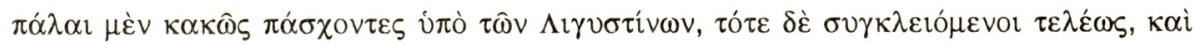

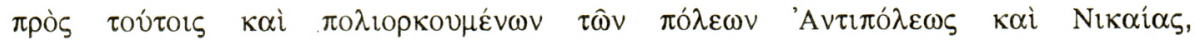

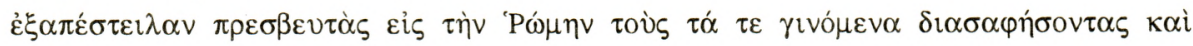

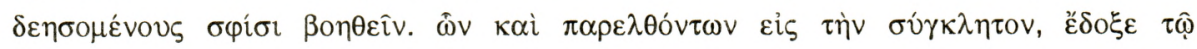

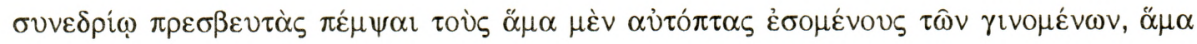

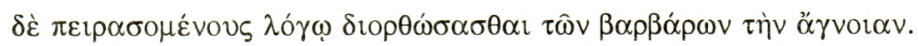

(8). Vers la même époque, arrivèrent des ambassadeurs envoyés par les Massaliotes. Ceux-ci supportaient depuis longtemps les attaques des Ligures et étaient maintenant bloqués, avec deux de leurs villes assiégées, Antipolis et Nikaia. Des ambassadeurs avaient été dépêchés pour raconter à Rome ce qui s'était passé et demander de l'aide. Le Sénat décida alors d'envoyer des légats pour examiner ce qui se passait et tenter de négocier afin de ramener les barbares à la raison.

Décider d'envoyer une mission pour régler diplomatiquement le problème était bien dans les habitudes du Sénat. En revanche, que Rome pénètre militairement sur la Côte d'Azur, voilà qui était nouveau. Trois consuls furent donc choisis pour tenter de ramener les Oxybiens et les Déciates à de meilleurs sentiments, et de les convaincre de l'intérêt qu'ils avaient à collaborer avec les civilisés.

Par un beau matin de l'an 599 de Rome, Flaminius, M. Popilius Laenas et L. Puppius embarquent donc pour la Gaule avec de nombreux serviteurs, mais aucun soldat, en vue de parlementer avec les barbares et d'obtenir qu'ils mettent fin à leurs coupables entreprises. Les ambassadeurs de Massalia, qui étaient venus se plaindre au Sénat, les accompagnent dans leur mission de paix.

Flaminius, M. Popilius Laenas et L. Puppius durent accepter ce voyage chez les barbares Ligures sans enthousiasme. Ils se souvenaient sans doute de la protestation de deux consuls à l'issue d'un tirage au sort qui venait de leur attribuer la Ligurie pour province : "C'est un affront que d'envoyer deux 
consuls dans les vallées ligures ! "(5) s'était exclamé l'un d'entre eux. Au bout de quatre ou cinq jours de navigation, les consuls voient au loin se profiler la 212 Côte d'Azur et émerger des eaux les îles de Léro et Lérina, appelées de nos jours Ste Marguerite et Ste Honorat.

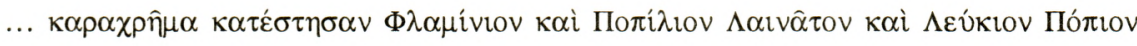

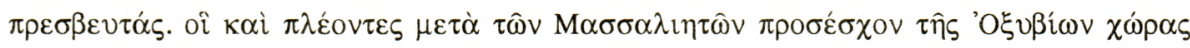

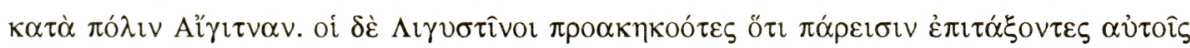

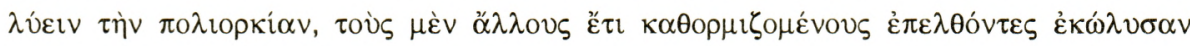

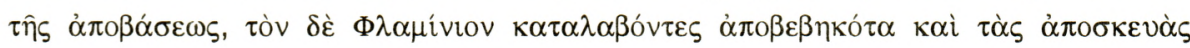

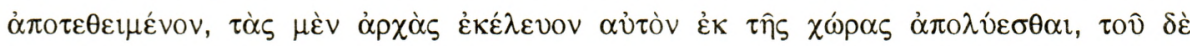

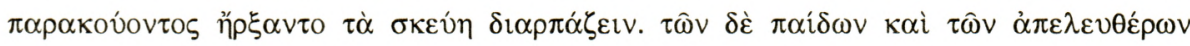

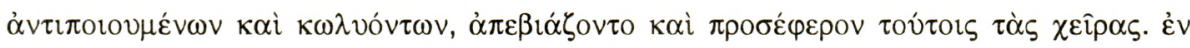

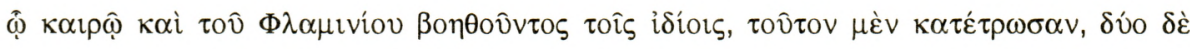

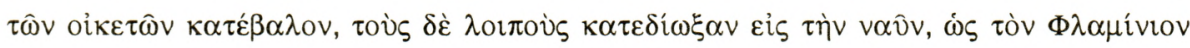

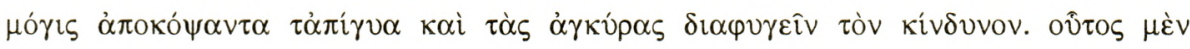

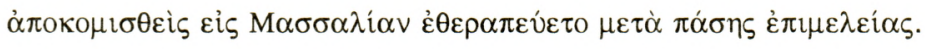

(9). ... Flaminius, Popilius Laenas et Lucius Pupius furent choisis. Ils embarquèrent avec les Massaliotes et abordèrent dans le territoire des Oxybiens, près de la cité d'Aegitna. Les Ligures avaient entendu dire qu'ils venaient pour leur ordonner de lever le siège; ils accoururent et empêchèrent de débarquer ceux qui n'avaient pas encore abordé, mais ils trouvèrent Flaminius qui avait déjà débarqué avec tous ses bagages. Ils commencèrent par lui enjoindre de quitter leur territoire, puis, devant son refus, à piller ses bagages. Ses esclaves et ses affranchis intervinrent alors pour les en empêcher, mais les Ligures se mirent à les molester et les, frapper. Flaminius vint à l'aide de ses hommes: les Ligures le blessèrent, tuèrent deux de ses serviteurs et chassèrent les autres jusqu'à leur navire. Flaminius échappa de justesse au danger en faisant couper les amarres et les câbles des ancres. Il fut rapatrié à Massalia, où il reçut tous les soins nécessaires.

Flaminius est donc transporté à Massalia. Bien entouré, il se remet rapidement de ses blessures, mais les soins qu'il reçoit n'atténuent pas sa rage. L'offense subie par les consuls, et surtout par lui, n'est pas de celles qu'on oublie en quelques semaines. Il proteste avec force auprès du Sénat.

(5) Georges Reymond, Monaco Antique, Publications de la Faculté des Lettres et Sciences Humaines de Nice, Coll. Méditerranée Antique et Moderne, Monaco, 1970, p. 84. 
L'armée doit réparer l'injure! Rome ne souhaitait pas la guerre, mais cette vexation va entraîner la Côte d'Azur dans une spirale tragique. Pour la première fois, les soldats romains franchissent le Var afin d'intervenir en Gaule.

\section{AEGITNA : LA BATAILLE}

Polybe raconte ainsi cette bataille :

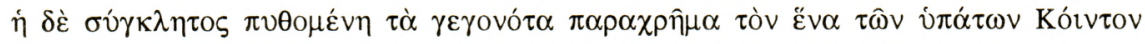

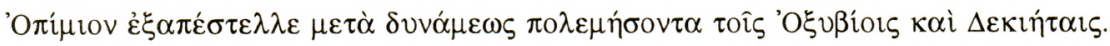

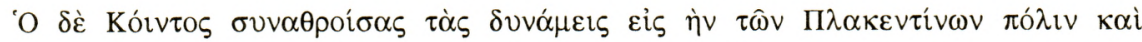

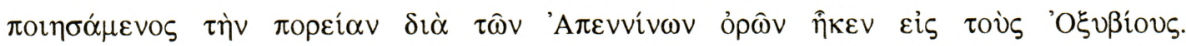

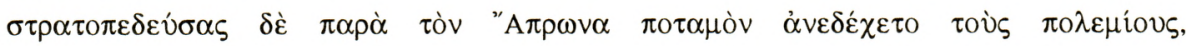

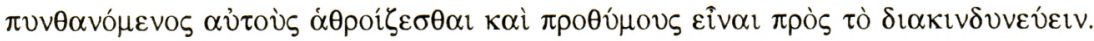

Lorsque le Sénat fut informé de ces événements, il envoya immédiatement un des deux consuls, Quintus Opimius Postumius, avec une armée pour faire la guerre aux Oxybiens et aux Déciates. (10). Postumius rassembla ses troupes à Placentia (Plaisance), traversa les monts Apennins et arriva chez les Oxybiens. Il campa son armée près du fleuve Apron, puis attendit les ennemis, ayant été informé qu'ils se rassemblaient, bien résolus à combattre.

Polybe ne nous dit pas par quelle voie Opimius s'est rendu chez les Oxybiens. Par terre ou par mer? La XXIIe légion venait de Plaisance, colonie romaine depuis 218 avant J.C où les consuls Lepidus et Flaminius avaient fait établir, vers 187 av J.C, une voie militaire depuis les environs de Rimini à travers les Apennins. C'est sans doute par cette voie nommée Emilia que, trente et un ans après sa création, Quintus Opimius conduisit ses soldats et franchit le Col de Tende. Ensuite, l'armée a pu continuer par voie terrestre et passer à proximité de Nikaïa et d'Antipolis ou contourner le territoire oxybien par le nord. Puis, ils auraient longé le cours de la Siagne, attaqué Cannes, poursuivi les Oxybiens vers Antibes et rencontré les Déciates à Golfe-Juan ou plus loin dans la Plaine de la Brague. D'autres auteurs estiment que les 
Romains, arrivés à pied par le Col de Tende, ont dû embarquer ensuite dans un port de la Ligurie contrôlé par les Romains, Vintimille ou plutôt Monaco.

Si, au contraire, Quintus Opimius arriva par la mer et fit embarquer son armée à Monaco, il a abordé ensuite un des points du golfe de La Napoule, près de l'embouchure de la Siagne sur les rives de laquelle il devait asseoir son camp. Cependant, compte tenu de l'importance des forces qu'il amenait, il a peut-être fait voile directement d'Italie.

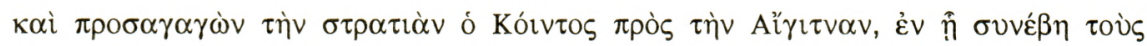

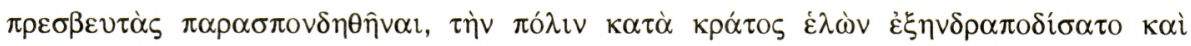

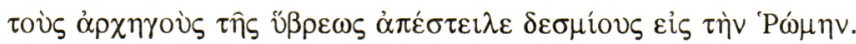

Quintus mena ensuite son armée devant Aegitna, où les légats avaient souffert du viol des conventions. Il prit la ville d'assaut, réduisit ses habitants à l'esclavage et envoya enchaînés à Rome les responsables de cette insulte.

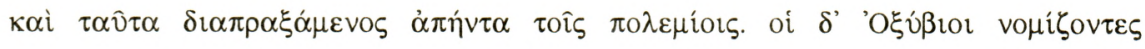

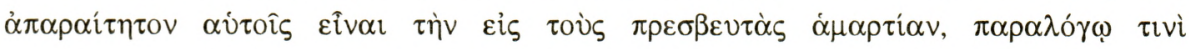

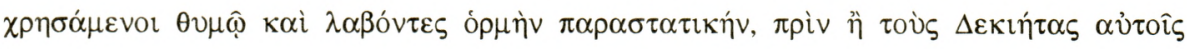

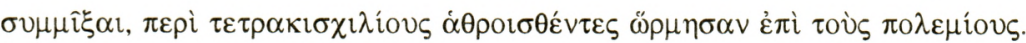

Cela fait, il marcha au-devant des ennemis. Les Oxybiens, convaincus que leur crime contre les légats était inexpiable, et saisis d'une singulière témérité, dans un assaut désespéré, sans attendre d'être rejoints par les Déciates, rassemblés en une force d'environ quatre mille hommes, se jetèrent sur leurs ennemis.

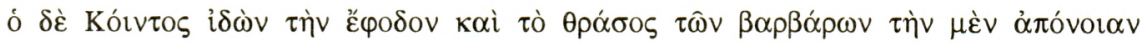

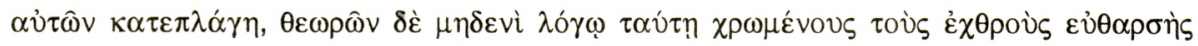

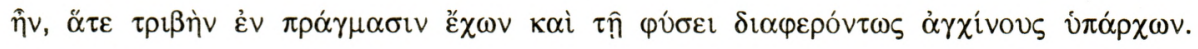

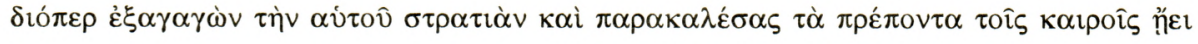

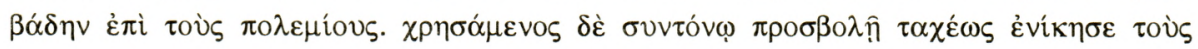

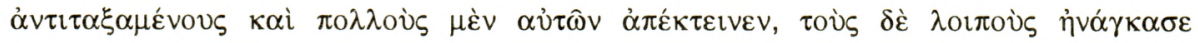

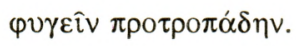

Quintus, devant l'offensive et l'impétuosité des barbares, fut frappé par cette inconscience, mais, constatant qu'elle ne s'appuyait sur aucune raison valable, il resta plein de confiance, en commandant plein d'expérience et particulièrement avisé. 
Il fit donc sortir son armée, l'exhorta dans les termes appropriés aux circonstances, et la fit avancer au pas à la rencontre de l'ennemi. Une violente attaque lui apporta ensuite une victoire rapide. Un grand nombre de ses adversaires furent tués et le reste forcé de fuir précipitamment.

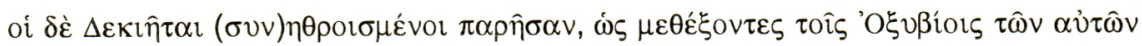

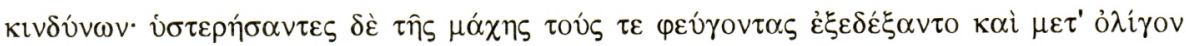

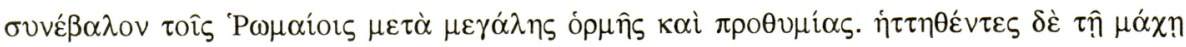

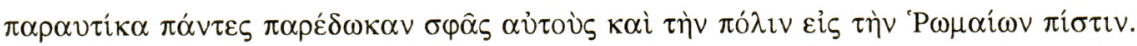

Les Déciates arrivèrent alors, avec toutes leurs forces, pour soutenir les Oxybiens, mais la bataille était terminée. Ils rallièrent cependant les fuyards, puis, chargèrent peu après avec beaucoup d'impétuosité et de courage. Défaits dans la bataille, aussitôt et sans conditions, ils se rendirent tous et remirent leur ville aux Romains.

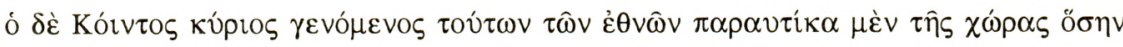

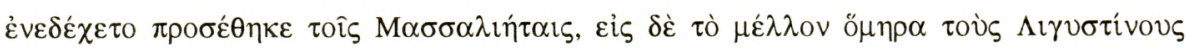

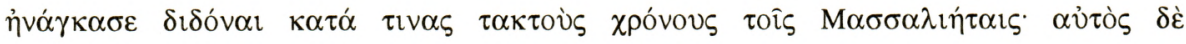

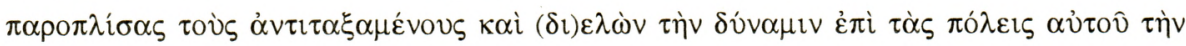

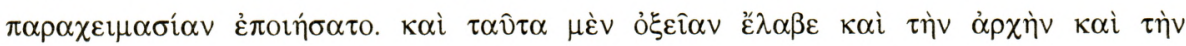
$\sigma v \vee \tau \dot{\varepsilon} \lambda \varepsilon \imath \alpha \nu$.

Quintus, devenu mâ̂tre de ces peuples, distribua aussitôt aux Massaliotes tous les territoires qu'il jugea bon et obligea pour l'avenir les Ligures à remettre des otages changés à date régulières. Il désarma les troupes adverses, répartit ses forces entre les villes et y prit ses quartiers d'biver. Cette campagne commença et finit ainsi rapidement.

Cette brève et terrible expédition romaine en faveur des comptoirs massaliotes, première du genre, ramène pour un temps la paix sur la Côte d'Azur. Libérées de l'entrave indigène, Antipolis et Nikaïa connaissent un long moment de répit. Mais c'est le calme avant la tempête, car la tranquillité retrouvée des cités grecques exaspère les peuplades d'alentour. En dépit de nombreuses pertes, les Oxybiens et les Déciates gardent l'espoir de parvenir à se débarrasser de leurs hôtes encombrants et des belliqueux Romains. Leur territoire, déjà exigu, a considérablement rétréci depuis la victoire de Quintus Opimius qui a entraîné la cession à Massalia d'une bande côtière de douze stades $(2.200 \mathrm{~m})$ de large du Var à l'Argens. Ce cordon de sécurité, posé par 
les Romains le long de l'ancien chemin ligure, porte un coup mortel aux Oxybiens et aux Déciates qui doivent quitter leurs ports respectifs et, pour les Oxybiens, abandonner leur capitale incluse dans l'espace attribué aux Massaliotes. Les deux tribus vaincues sont obligées de s'éloigner des rivages et de chercher d'autres ressources que la pêche et la piraterie. Ils s'installent dans l'arrière-pays grassois et, de pêcheurs et pirates, deviennent agriculteurs.

Le deuxième siècle avant Jésus-Christ s'achève. Massalia et ses colonies, sauvées d'une invasion barbare, ont encore quelques décennies ensoleillées devant elles. Mais, sans le savoir, elles sont déjà engagées sur un doux déclin. Les comptoirs de la Côte d'Azur, agrandis aux dépens des tribus ligures, n'appartiennent plus vraiment aux Grecs. La route terrestre, ainsi que le commerce vers la Gaule qui avait justifié leur implantation, passent à présent sous le contrôle romain.

\section{LA LOCALISATION D'AEGITNA}

La lecture du texte de Polybe ne nous avance pas sur l'emplacement de la bataille d'Aegitna. La donnée du problème tient dans la conjonction d'un fleuve, l'Apron, et d'une bourgade portuaire oxybienne. À cet énoncé, il faut ajouter un espace terrestre permettant le passage des cinq à six mille soldats de la XXIIe légion romaine, et leur rencontre avec quatre mille Oxybiens. Le mystère de l'emplacement de la bataille d'Aegitna a, depuis des siècles, épuisé tous ceux qui ont tenté de l'éclaircir. Il a provoqué des querelles passionnées d'historiens, chacun ayant son idée bien arrêtée sur le problème, le plus souvent par préférence sentimentale ou civique. Aegitna était un port, celui des Oxybiens, Polybe a au moins été clair sur ce point. Mais on ne connaît pas les limites exactes du territoire des Oxybiens et de celui des Déciates. C'est un autre motif de discorde entre les historiens. 


\section{a) Le fleuve Apron}

La bataille d'Aegitna s'est jouée quelque part entre le Var et l'Argens, ce qui laisse une grande latitude. Néanmoins, on doit exclure l'Argens ou le Reyran, car ces deux cours d'eau débouchent sur le territoire des Suelteri. Or, le port des Oxybiens, en toute logique, ne pouvait se trouver que sur le territoire de ces derniers. En outre, l'Argens et le Reyran, rivières des environs de Fréjus, sont très éloignées de Nikaïa et d'Antipolis que les Romains voulaient délivrer de l'entrave ligure. L'Apron ne peut donc être ni l'Argens ni le Reyran. Quant au Var, il était bien connu des Grecs qui l'appelaient l'Ouaros, et c'est forcément ce nom qu'aurait employé Polybe pour le désigner.

Si l'on élimine ces trois fleuves, il ne reste sur la Côte d'Azur que la Siagne, pouvant être perçue comme un fleuve, et quelques ruisseaux. De nombreux auteurs modernes ont cru reconnaître dans l'Apron, le Loup, la Brague ou encore la Cagne. De nos jours, ce ne sont que des ruisselets, et encore, en hiver seulement, car en été, ils ne contiennent qu'un filet d'eau, quand ils ne sont pas complètement asséchés. Or, la bataille d'Aegitna s'est sans doute déroulée à la fin de l'été ou au début de l'automne. En effet, après sa rapide victoire, Opimius et ses soldats ont hiverné sur la Côte d'Azur ainsi qu'il était habituel à une époque où l'on ne prenait la mer qu'aux beaux jours.

Les historiens qui n'admettent pas l'identification de l'Apron avec la Siagne avancent qu'à l'époque, le débit des cours d'eau était beaucoup plus important que de nos jours, mais il ne l'était sans doute pas assez pour faire un fleuve de ruisseaux tels que le Loup, la Brague ou la Cagne, à sec les trois quarts de l'année. Certes, la préposition « $\pi \alpha \rho \alpha$ » utilisée par Polybe pour situer le camp romain par rapport à l'Apron, signifie aussi bien "près de" que «aux environs de» ou "au-delà de», ce qui laisse la possibilité de l'établissement des Romains ailleurs que sur le bord du fleuve. Le combat aurait alors pu avoir lieu à bonne distance de l'Apron, Opimius, selon Polybe, s'étant porté vers 
l'ennemi et, par conséquent, ayant pu l'affronter ailleurs que sur les rives du fleuve.

Il reste que les trois petites rivières précitées coulent entre Nikaïa et Antipolis, c'est-à-dire en plein territoire des ennemis de Rome. Aussi semblerait-il curieux qu'Opimius, présenté par Polybe comme un général de grande expérience, ait imprudemment placé son camp au beau milieu des tribus qu'il venait combattre. Les indigènes qui tenaient Antipolis n'auraient pas manqué d'apercevoir les Romains et, sans doute, ne les auraient-ils pas laissés s'installer tranquillement à côté d'eux, sans intervenir.

En revanche, si l'Apron de Polybe est la Siagne, les collines de Vallauris auraient occulté le camp romain, le plaçant hors de vue des Ligures qui serraient de près Antipolis, et cette configuration aurait laissé à Opimius un espace suffisant pour le mouvement stratégique de ses nombreux soldats. Dans ce cas, la célèbre bataille aurait eu lieu dans la plaine de Laval, morne plaine qui, depuis les rives de la Siagne, s'étend vers Cannes. Mais rien n'est acquis sur ce point, et l'énigmatique fleuve de Polybe continue à ne charrier que des hypothèses.

b) La bourgade portuaire

Polybe nous informe qu'Opimius conduisit son armée devant Aegitna où le droit des ambassadeurs de Rome avait été violé. Aegitna était un port. On ne peut en douter puisque les trois malchanceux consuls, venus parlementer avec les Oxybiens, étaient arrivés par la mer, et que Flaminius, pour son malheur, y a débarqué avec ses serviteurs. Le récit de Polybe élimine, a priori, les villes situées loin du littoral, telles que les Encourdoules qui couronnent un mamelon à trois kilomètres du rivage, ainsi que d'autres agglomérations comme Mougins, Biot ou Villeneuve-Loubet, éloignées de la mer. Néanmoins, ces bourgades perchées pouvaient avoir leur port sur la côte la plus proche.

Aegitna a longtemps été donnée pour le berceau de Cannes. Le premier à avoir émis cette opinion était un géographe de Dantzig nommé Philipp 
Kluwer, né en 1580, mort en 1623. De nombreux auteurs ont suivi et confirmé par de nouvelles observations l'opinion de Kluwer. L'Apron était l'ancêtre de la Siagne. Le compte rendu de Polybe s'en trouvait éclairci alors que tout devenait brumeux en plaçant Aegitna à Biot ou à Villeneuve-Loubet. Mais Aegitna était un port alors qu'à l'époque des événements, Cannes n'offrait qu'une rade sablonneuse. Au IIe siècle avant Jésus-Christ, on naviguait dans des bateaux à voiles et à rames de plusieurs centaines de tonneaux pour lesquels il fallait envisager autre chose que le tirage à terre sur les plages. Faute de pouvoir accoster, des barques transportaient à terre les passagers des navires ancrés dans la rade.

Au reste, le port d'Aegitna n'était pas considérable. Strabon, se référant à Poseidonios, écrit (livre IV, 1, 6-4) : "En ce qui concerne les ports, enfin, seuls sont importants Fréjus et Massalia. Les autres sont de petite dimension; citons celui d'Oxybios auquel les Ligyens Oxybiens ont donné leur nom."

Cependant, en 1901, des fouilles dans l'étang de Vaugrenier, alimenté par la Brague, mirent à jour un trophée brisé en treize morceaux et décoré d'armes gauloises. On décida, un peu vite, qu'il commémorait la bataille d'Aegitna. Dès lors, l'opinion se détourna brusquement de Cannes pour se porter dans la plaine de la Brague, sur la commune de Villeneuve-Loubet, à côté d'Antipolis. Le port des Oxybiens se trouvait là, dans l'étang de Vaugrenier, et il communiquait avec la mer par un étroit goulet depuis longtemps disparu. D'ailleurs, une vieille histoire locale évoque des fantômes ligures hantant l'étang de Vaugrenier dans lequel une ville entière fut jadis engloutie !

Après la découverte du trophée de la Brague, des fouilles entreprises sur la Colline San Andrieu, une butte voisine de l'étang de Vaugrenier, baignée à sa base par la rivière du Loup, ont révélé une occupation humaine antique aux alentours du IIe siècle avant notre ère, puis des restes d'incendie et, pour finir, une réoccupation du site par les Romains. On en a conclu, peut-être imprudemment, que la capitale des Oxybiens était perchée à cet endroit, sur 
un oppidum, dans la commune de Villeneuve-Loubet. Quant à l'Apron, c'était le Loup... Par ailleurs, Jean-Edouard Dugand ${ }^{(6)}$ a situé Aegitna sur le piton conique des Hauts-de-Cagnes, mais le trophée de la Brague est bien postérieur à la bataille d'Aegitna. On le donne pour contemporain des monnaies Lépides frappées en 42 avant notre ère, aussi peut-on difficilement admettre qu'il ait été élevé afin de rappeler la victoire de Quintus Opimius cent douze ans après qu'elle a eu lieu! Sans compter que Opimius, mort depuis longtemps, n'avait pas eu droit de son vivant aux honneurs du triomphe à Rome et avait dû se contenter de l'entrée solennelle à Rome, l'ovatio.

Une autre tradition qui se conserve à Vallauris Golfe-Juan veut qu'il y ait eu un port ligure dans l'actuelle commune et que ce port ait été celui d'Aegitna dont la partie habitée occupait le site perché des Encourdoules, mais ce nom, "Cordula» au Moyen-Âge, n'a aucun rapport avec celui d'Aegitna, et surtout, si le port d'Aegitna s'était trouvé à Golfe-Juan, les Oxybiens n'auraient pas laissé les Romains massacrer ou charger de chaînes les vieillards, les femmes, et les enfants sans se porter aussitôt à leur secours. Il semble qu'il faille chercher ailleurs qu'à Golfe-Juan l'emplacement d'Aegitna. À Mougins prétendent certains : après la cession du territoire côtier, cadeau de Quintus Opimius aux Massaliotes à l'issue de la bataille, les habitants de Cannes obligés de s'éloigner de la mer à la distance de douze stades, se seraient retranchés à Mougins, juste au-dessus de Cannes, dans les limites imposées. Établis sur les hauteurs de leur bourgade perdue, les Oxybiens auraient donné à leur nouvelle ville le nom de celle qu'ils avaient abandonnée : la montagne d'Aegitna, Mons Aegitna, dont on aurait fait plus tard Mongins, puis Mougins aujourd'hui.

\footnotetext{
(6) De l'Aegitna de Polybe au trophée de la Brague, Publications de la Faculté de Lettres et de Sciences Humaines de Nice, Les Belles Lettres, Monaco, 1970
} 
Comme on le voit, le problème est loin d'être résolu. En attendant des fouilles miraculeuses, Aegitna continue à flotter dans les limbes de l'histoire antique et l'Apron à couler, tel le Styx, dans les ténèbres...

\section{LA FIN DES GRECS SUR LA CÔTE D'AZUR}

Le premier siècle est arrivé et le crépuscule descend, tranquillement, sur les comptoirs massaliotes de la Côte d'Azur. La paix romaine approche, mais Massalia, l'alliée fidèle de Rome, va connaître des jours noirs et, une dernière fois, Antipolis et Nikaïa vont tenir, à ses côtés la place de dévoués compatriotes avant la séparation définitive, qui commencera en 49 avant Jésus-Christ avec l'arrivée de Jules César à Nikaïa (ou à Antipolis).

Le passé de la Côte d'Azur contient l'héritage des Grecs, mais il est difficilement saisissable, car il ne repose pas sur une réalité matérielle. Pas un rempart, pas un mur, pas un monument n'a résisté. Mais la Côte d'Azur était déjà bien entrée dans le monde civilisé antique avant la conquête romaine, frottant ses éléments à la culture des Grecs et à celle d'autres civilisations qui lui avaient rendu visite: Phéniciens, Étrusques, Rhodiens... cette terre de confluences a mûri son génie au rayonnement des uns et des autres.

C'est peut-être la raison pour laquelle, sur la Côte d'Azur, malgré une assimilation rapide, les indigènes ne seront jamais totalement tributaires des Romains sur le plain culturel, car ils ont été hellénisés avant d'être romanisés. En fait d'assimilation, il s'agit plutôt d'une symbiose entre des Ligures hellénisés et des Romains dont la Grèce a été l'éducatrice.

À présent, au milieu du tohu-bohu de la "french Riviera ", on n'entend plus la voix du bel héritage. Pourtant, dans ce cadre dévoré de lumière, envahi par le bleu éblouissant de la Méditerranée, les Grecs continuent à déclamer, en silence, l'histoire d'un demi-millénaire de leur présence sur la Côte d'Azur. 